\title{
Numerical investigation of the stability of the rational solutions of the nonlinear Schrödinger equation
}

\author{
A. Islas and C.M. Schober \\ Department of Mathematics, University of Central Florida, Orlando, FL 32816, USA
}

\begin{abstract}
The rational solutions of the nonlinear Schrödinger (NLS) equation have been proposed as models for rogue waves. In this article we develop a highly accurate Chebyshev pseudospectral method (CPS4) to numerically study the stability of the rational solutions of the NLS equation. The scheme CPS4, using the map $x=\cot \theta$ and the FFT to approximate $u_{x x}$, correctly handles the infinite line problem. A broad numerical investigation using CPS4 and involving large ensembles of perturbed initial data, indicates the Peregrine and second order rational solutions are linearly unstable.

Although standard Fourier integrators are often used in current studies of the NLS rational solutions, they do not handle solutions with discontinuous derivatives correctly. Using standard Fourier pseudo-spectral method (FPS4) for Peregrine initial data yields tiny Gibbs oscillations in the first steps of the numerical solution. These oscillations grow to $\mathcal{O}(1)$, providing further evidence of the instability of the Peregrine solution. To resolve the Gibbs oscillations we modify FPS4 using a spectral-splitting technique which significantly improves the numerical solution.
\end{abstract}

Keywords: rogue waves, Peregrine solution, stability, spectral splitting, Chebyshev spectral methods, spectral methods

\section{Introduction}

One possible mechanism for rogue wave generation is the nonlinear focusing of waves due to the modulational instability. As a result, rogue waves are often studied in the framework of the focusing nonlinear Schrödinger (NLS) equation,

$$
i u_{t}+u_{x x}+2|u|^{2} u=0
$$

Certain solutions of the NLS equation exhibit the distinguishing features of rogue waves, i.e. transience, localization, and steepness, and have emerged as prototypes for rogue waves. On a periodic domain one such class of solutions is the family of multi-mode heteroclinic orbits over unstable plane waves with $\tilde{N}$ unstable modes (UMs) [10, 4]. These heteroclinic orbits, with $M \leq \tilde{N}$ modes excited, are also referred to as $M$ mode spatially periodic breathers (SPBs). On the infinite line a hierarchy of rational solutions of the NLS equation can be derived using a modified Darboux transformation and used to model rogue waves [1]. The 
lowest order rational solution, the Peregrine solution, can also be obtained as a singular limit of the one mode SPB as the spatial period becomes unbounded [11].

In our earlier studies we examined: 1) the stability of the SPBs with respect to perturbations of initial data and 2) the persistence of such structures in more realistic higher order NLS models. Via the squared eigenfunction approach it was analytically established that the SPBs are typically unstable [5]. Only the "maximal" SPBs (i.e. those with $\tilde{N}$ spatial modes over a background with $\tilde{N}$ instabilities) are neutrally stable and thus most likely to be reproducible in an experimental setting [5]. Further, the stable coalesced two-mode SPB was shown to persist in higher order NLS models $[4,9]$.

The robustness of the rational solutions has been examined in $[13,18,20]$. In careful studies a reasonable wave evolution with persistence of general features, such as the maximum wave height, is obtained for a finite time within some finite domain [21, 22]. While the robustness of the Peregrine solution is the primary concern in these studies, the question of stability is a different issue which specifically addresses whether perturbations stay close in an appropriate mathematical sense.

The stability of the rational solutions is an open question. In this article we numerically investigate the linear stability of the first and second order rational solutions, $p_{i}(x, t)$, with respect to a wide range of random perturbations $f_{j}(x)$ typically encountered in experimental settings. To do so, in Section 3 we develop a highly accurate Chebyshev pseudo-spectral (CPS4) method that uses the map $x=\cot \theta$ in combination with the Fast Fourier Transform to approximate $u_{x x}$ on an infinite domain. The number of Chebyshev points and the time step are chosen so that the maximum numerical error over the time frame is $\mathcal{O}\left(10^{-7}\right)$ ), considerably smaller than the size of the perturbations $f_{j}\left(\mathcal{O}\left(10^{-2}\right)\right)$. In Section 4 , for both the first and second order rational solutions, and for each $f_{j}(x)$, an ensemble of 100 numerical experiments are carried out varying the random component in the initial data. All of the perturbed solutions rapidly diverge from the corresponding rational solution $p_{i}(x, t)$ indicating the rational solutions are unstable, having inherited the instabilities of the underlying plane wave. Given the interpretation of the rational solutions as singular limits of the SPBs, this is consistent with the instability results for the SPBs.

In current studies of the rational solutions, standard Fourier methods are widely used (with a very large number $N$ of Fourier modes, e.g. for spatial period $L=1000, N=65,536$ ) without a discussion of the related numerical errors (see e.g. $[1,6,13,16,18]$ ). In Section 5 we discuss the errors arising when using Fourier spectral methods with Peregrine initial data, in order to understand and mitigate them. We provide an integrator which can treat initial data with discontinuous derivatives over periodic domains, e.g. it accurately resolves the Peregrine solution with far fewer Fourier modes $(L=800, N=8192)$.

The truncated and periodically extended Peregrine initial data, $P(x, 0)$, has imperceptible corners at the boundary resulting in small jump discontinuities in the derivatives. Using a standard pseudo-spectral scheme (FPS4) the Fourier approximations of $P_{x}(x, 0)$ and $P_{x x}(x, 0)$ exhibit Gibbs oscillations $\left(\mathcal{O}\left(10^{-6}\right)\right)$. The oscillations in $P(x, t)$ grow exponentially to $\mathcal{O}(1)$ providing further evidence of the instability of the Peregrine solution.

In Section 5.4 we modify FPS4 using a spectral-splitting technique due to Gottlieb and 
Shu $[19,15]$. The Fourier modes associated with the Gibbs oscillations in $P(x, t)$ are removed and only those modes that contain the critical information remain, significantly improving the numerical solution. We note that these new integrators are not limited to the situation at hand, i.e. to truncated rational solutions. Rather, the modified FPS4 method can be used to address the evolution of more general piecewise continuous initial data with discontinuous derivatives anywhere within the spatial domain. The Chebyshev pseudo-spectral method is potentially applicable to whole line problems with slowly decaying initial data and can be adapted for other equations.

\section{Analytical Background}

The NLS equation (1) is a completely integrable Hamiltonian system and can be interpreted as the solvability condition of the following Zakharov-Shabat linear system (Z-S) [17]:

$$
\left.\begin{array}{rl}
\mathcal{L}^{(x)} \mathbf{v} & =\left(\begin{array}{cc}
\frac{\partial}{\partial x}+i \lambda & -u \\
u^{*} & \frac{\partial}{\partial x}-i \lambda
\end{array}\right) \mathbf{v}=0 \\
\frac{\partial}{\partial t}-i\left(|u|^{2}-2 \lambda^{2}\right) & -i u_{x}-2 \lambda u \\
-i u_{x}^{*}+2 \lambda u^{*} & \frac{\partial}{\partial t}+i\left(|u|^{2}-2 \lambda^{2}\right)
\end{array}\right) \mathbf{v}=0,
$$

where $\lambda$ is the spectral parameter and $u(x, t)$ is a solution of the NLS equation itself.

The SPBs and rational solutions of the NLS equation exhibit rogue wave features since they are intimately connected to the modulational instability of the Stokes wave solution

$u_{a}(t)=a \mathrm{e}^{2 i a^{2} t}$. The stability of $u_{a}(t)$ can be examined by considering perturbations of the form $u(x, t)=u_{a}(x, t)+\epsilon(x, t)$ and linearizing for small $\epsilon$. For periodic boundary conditions, $u(x, t)=u(x+L, t)$, one assumes a Fourier series representation with modes $\epsilon_{j}(x, t) \propto e^{i\left(k_{j} x+\sigma_{j} t\right)}, k_{j}=2 \pi j / L$, which gives the following criterion for linear instability: if $0<\left(\frac{\pi j}{L}\right)^{2}<a^{2}, j \in \mathbb{N}$, then the $j$-th mode is unstable and $u_{a}(t)$ has $\tilde{N}=\lfloor a L / \pi\rfloor \operatorname{UMs}(\tilde{N}$ is the greatest integer less than or equal to $a L / \pi)$. For $-\infty<x<\infty$, using Fourier transforms to solve the linearized NLS, one finds $u_{a}(t)$ is unstable since $\epsilon(x, t)$ grows exponentially for all wavenumbers $k$ such that $|k|<2 a$.

\subsection{Spatially periodic breather (SPB) solutions}

On a periodic domain, the $\tilde{N}$ UMs of the plane wave, the "seed" solution, correspond to $\tilde{N}$ degenerate complex discrete spectra $\lambda_{j}^{d}$ of the associated Zakharov-Shabat linear system. Explicit formulas for the $M$-mode SPBs over a plane wave with $\tilde{N} \geq M$ UMs require $M$ iterations of the Bäcklund-gauge transformation for the NLS equation [12]. A single Bäcklund transformation at complex $\lambda_{j}^{d}$ generates a single mode SPB corresponding to the $j$-th UM of $u_{a}(t)$. The single mode SPB associated with the first $(j=1) \mathrm{UM}$ is

$$
b_{1}(x, t)=a e^{2 i a^{2} t}\left(\frac{1+2 \cos k_{1} x e^{\sigma t+\gamma} e^{2 i \phi}+\sec ^{2} \phi e^{2(\sigma t+\gamma)} e^{4 i \phi}}{1+2 \cos k_{1} x e^{\sigma t+\gamma}+\sec ^{2} \phi e^{2(\sigma t+\gamma)}}\right)
$$

where $k_{1}=2 \pi / L, \sigma=k_{1} \sqrt{4 a^{2}-k_{1}^{2}}, \phi=\sin ^{-1}\left(k_{1} / 2 a\right), L$ is the period, $a$ is the amplitude of the background plane wave, and $\gamma$ is the temporal phase parameter $[10,4,2]$. Figure $1(\mathrm{a})$ shows the surface of $\left|b_{1}(x, t)\right|$ for $a=0.5, L=40$, and $\gamma=0$. 

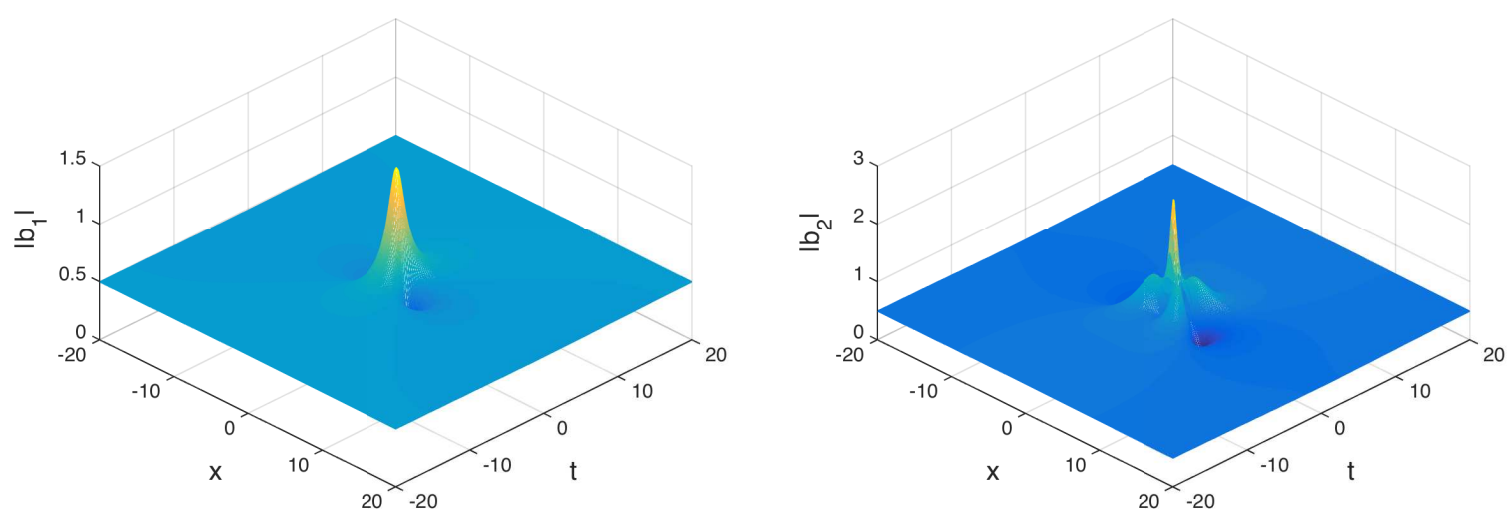

Figure 1: Surfaces for (a) the single mode SPB $\left|b_{1}(x, t)\right|$ and (b) the two mode SPB $\left|b_{2}(x, t)\right|$ with a coalesced peak.

Successive applications of the Bäcklund-gauge transformation at complex $\lambda_{1}^{d}$ and $\lambda_{2}^{d}$ generates a two mode SPB family $b_{2}(x, t)$ (for the explicit formulas see e.g. [5]). Figure 1(b) shows the amplitude $\left|b_{2}(x, t)\right|$ of a special member of the family of two-mode SPBs, where the two modes have "coalesced" into a single coherent structure (for $a=0.5$ and $L=40$ ).

An important feature of the $M$-mode SPBs is their localization in time: the rogue wave structure emerges only once due to nonlinear focusing while as $t \rightarrow \pm \infty$ the SPBs exponentially decay to a phase translate of the plane wave. As $L$ increases the SPBs become effectively localized in space.

\subsection{Rational solutions}

For whole-line non-zero boundary conditions, the main tool for constructing rational solutions is a modified Darboux transformation [1]. However, the Peregrine solution, the lowest order rational solution of the NLS equation, is derivable as a singular limit of the SPB $b_{1}(x, t)$ as the spatial period $L \rightarrow \infty$ [6]. The Peregrine solution of the NLS equation (1) is given by

$$
p_{1}(x, t)=a e^{2 i a^{2} t}\left[1-\frac{4\left(1+4 i a^{2} t\right)}{1+16 a^{4} t^{2}+4 a^{2} x^{2}}\right],
$$

where $a$ is the amplitude of the background plane wave. Although the higher order rational solutions can similarly be derived as the limit of the $\mathrm{SPBs} b_{i}(x, t)$ as $L \rightarrow \infty$, it is easier to explicitly construct solutions of the NLS equation as quotients of Wronskians of order $2 \tilde{N}$ and take the limit as all the periods approach infinity [7]. In particular, the exact second 
order rational solution is given by

$$
\begin{aligned}
p_{2}(x, t)= & a e^{2 i a^{2} t} \frac{G(x, t)}{H(x, t)} \\
G(x, t)= & 64 a^{6} x^{6}+\left(768 a^{4} t^{2}-144-768 i t\right) a^{4} x^{4} \\
& +\left(3072 a^{8} t^{4}-5760 a^{4} t^{2}-6144 * i a^{6} t^{3}+1152 i a^{2} t-180\right) a^{4} x^{2} \\
& +45-1536 i a^{6} t^{3}-1872 a^{4} t^{2}-8448 a^{8} t^{4}+4096 a^{12} t^{6}+720 i a^{2} t-12288 i a^{10} t^{5}, \\
H(x, t)= & 64 a^{6} x^{6}+\left(768 a^{4} t^{2}+48\right) a^{4} x^{4}+\left(-1152 a^{4} t^{2}+108+3072 a^{8} t^{4}\right) a^{2} x^{2} \\
& +4096 a^{12} t^{6}+6912 a^{8} t^{4}+1584 a^{4} t^{2}+9 .
\end{aligned}
$$

The surfaces of $\left|p_{1}(x, t)\right|$ and $\left|p_{2}(x, t)\right|$ (for $a=0.5$ ) are visually indistinguishable from those of the periodic SPBs for $-L / 2<x<L / 2$, as seen in Figure 1(a-b), respectively (but

differ outside this interval). Although the rational solutions are localized in both space and time they decay slower to the plane wave than their exponentially decaying SPB counterparts: $p_{i}(x, t)$ decay as i) $t^{-1}$ as $t \rightarrow \pm \infty$ and ii) as $x^{-2}$ as $x \rightarrow \pm \infty$.

\section{Chebyshev pseudo-spectral method}

Since the rational solutions decay algebraically as $x \rightarrow \pm \infty$, we develop a highly accurate Chebyshev pseudo-spectral method to numerically integrate the NLS equation. The unbounded domain is treated by combining the Chebyshev points $y=\cos \theta$, which take $\theta \in[0, \pi]$ to $y \in[-1,1]$, combined with the map

$$
x=\frac{y}{\sqrt{1-y^{2}}}
$$

which, in turn, takes the interval $(-1,1)$ to the infinite interval $(-\infty, \infty)[3]$. The composition of these maps gives

$$
x=\frac{\cos \theta}{\sin \theta}=\cot \theta, \quad \theta \in(0, \pi) .
$$

An advantage of this approach is that the derivatives of a function $f(x)$ can be approximated using the Fast Fourier Transform (FFT) routine [14]. Let $f(x) \approx F(\theta)$ be the trigonometric interpolant of the evenly extended $\left\{f_{j}\right\}$ data. That is, let

$$
f(x) \approx F(\theta)=\frac{1}{2 \pi} \sum_{k=-N+1}^{N} \hat{f}_{k} e^{i k \theta}=\sum_{n=0}^{N} a_{n} \cos n \theta .
$$

The first derivative is then given by

$$
f^{\prime}(x) \approx \frac{F^{\prime}(\theta)}{d x / d \theta}=-\sin ^{2} \theta F^{\prime}(\theta)=G(\theta)
$$

while the second derivative is given by

$$
f^{\prime \prime}(x) \approx-\sin ^{2} \theta G^{\prime}(\theta)=\sin ^{2} \theta\left(2 \sin \theta \cos \theta F^{\prime}(\theta)+\sin ^{2} \theta F^{\prime \prime}(\theta)\right)
$$


The derivatives $F^{\prime}(\theta)$ and $F^{\prime \prime}(\theta)$ can be obtained by multiplying $\hat{f}$ by $i k$ and $-k^{2}$, respectively, and then applying the inverse Fast Fourier Transform IFFT routine. The other factors can be expressed in terms of $x$ yielding the following approximation for the second derivative:

$$
f^{\prime \prime}(x) \approx \frac{2 x F^{\prime}(\theta)+F^{\prime \prime}(\theta)}{\left(1+x^{2}\right)^{2}} .
$$

Let $U(t)=\left(U_{1}(t), U_{2}(t), \ldots, U_{N}(t)\right)$ be a discretization of $u(x, t)$ at the grid points $X=\left(x_{1}, x_{2}, \ldots, x_{N}\right)$ given by the map (6). Using (7) to approximate the second partial derivative, a numerical solution of the NLS equation can be obtained by using a 4th-order Runge-Kuta scheme in time to solve the system of complex ordinary differential equations

$$
U_{t}=i\left(U_{x x}+2|U|^{2} U\right)
$$

We denote this 4th-order in time Chebyshev pseudo-spectral scheme for solving the NLS equation as CPS4.

\subsection{Accuracy of the $\mathrm{CPS}_{4}$ scheme}

In this subsection we examine the accuracy of the Chebyshev pseudo-spectral scheme in resolving the Peregrine and higher order rational solutions of the NLS equation, demonstrating that CPS4 is appropriate for numerically investigating the stability of these solutions.

Let $P_{i}(x, t)$ be the numerical solutions corresponding to initial data $P_{i}(x, 0)=p_{i}(x, 0)$ for $i=1,2$ (see equations (4)-(5) for the exact rational solutions). The errors, $E_{i}(t)$, in the numerical approximation of the rational solutions, for each $t$, are computed by

$$
E_{i}(t)=\max _{x}\left(|| p_{i}(x, t)|-| P_{i}(x, t) \|\right)
$$

Figures 2(a) and 3(a) show the errors $E_{i}(t)$ obtained with CPS4 for $N=256 \mathrm{~m}$ Chebyshev nodes, where $m=1, \ldots, 4$, and corresponding time steps $d T \approx 2 \times 10^{-5} / m^{2}$, respectively. The surfaces $\left|P_{i}(x, t)\right|$ of the numerical solutions $(N=1024)$ are displayed for $-10 \leq x \leq 10$, $0 \leq t \leq 15$ in Figures 2(b) and 3(b), respectively. Here the solutions are displayed over a small spatial domain to illustrate that the main structures are well resolved. For large $x$, the numerical solutions decay appropriately to the plane wave and do not exhibit any irregularities.

When $N=1024$ and $d T=1.25 \times 10^{-6}$ the $\max _{t \in[0,15]} E_{i}$ is $\mathcal{O}\left(10^{-7}\right)$. Further, the global invariants, the norm $\mathcal{I}=\int_{\mathcal{D}}|u|^{2} \mathrm{~d} x$ and Hamiltonian $\mathcal{H}=-i \int_{\mathcal{D}}\left\{\left|u_{x}\right|^{2}-|u|^{4}\right\} d x$ are preserved $\mathcal{O}\left(10^{-8}\right)$. As a result $N=1024$ and $d T=1.25 \times 10^{-6}$ are used in the subsequent numerical study of stability of the rational solutions. Setting $\theta=(n-1) \pi /(N-1), n=$ $1, \ldots, N$ in map (6), and replacing the end points at infinity, $x_{1}$ and $x_{N}$, with the finite linear extensions $2 x_{2}-x_{3}$ and $2 x_{N-1}-x_{N-2}$, respectively, $N=1024$ yields a computational spatial domain $L \approx 977$. However, the solution is often displayed over smaller spatial domains to enable close-ups of features of the solution. 

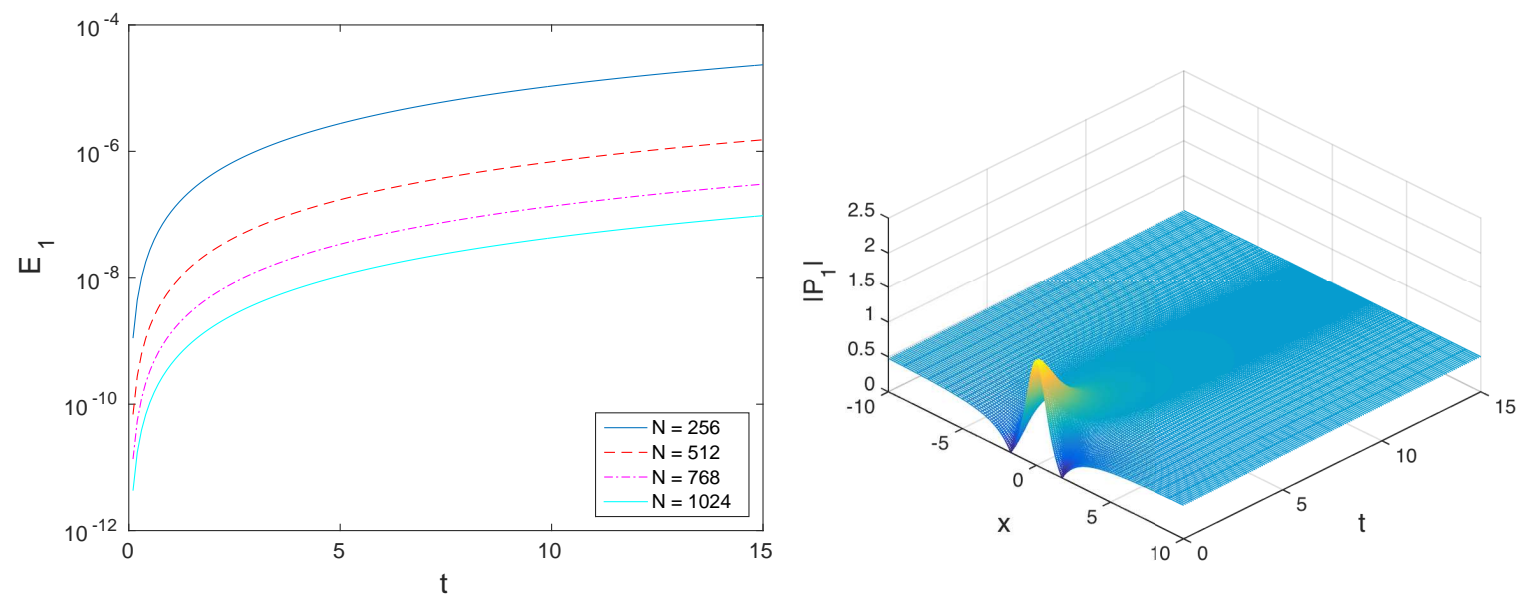

Figure 2: The numerical Peregrine solution obtained using CPS4: (a) the error $E_{1}(t)$ obtained using $N=$ $256, \ldots 1024$ Chebyshev nodes, and (b) $\left|P_{1}(x, t)\right|$.
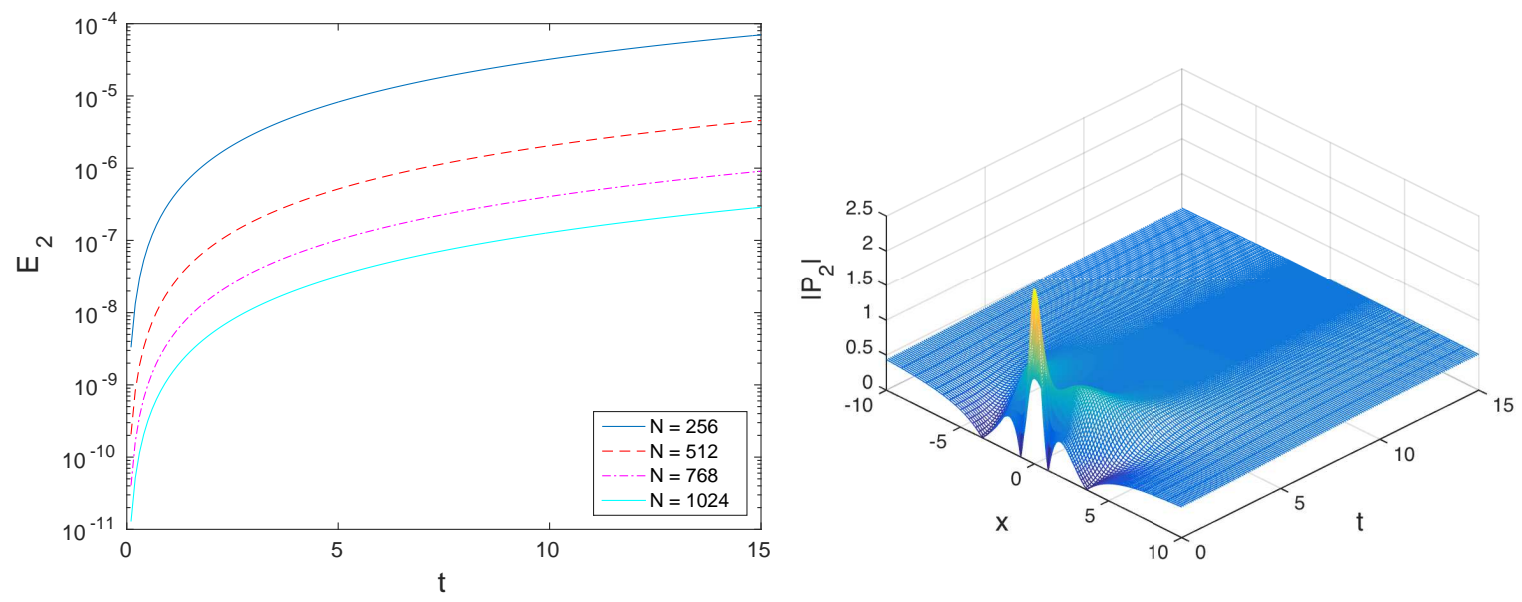

Figure 3: The numerical 2nd order rational solution obtained using CPS4: (a) the error $E_{2}(t)$ obtained using $N=256, \ldots 1024$ Chebyshev nodes, and (b) $\left|P_{2}(x, t)\right|$

\section{Numerical evidence of instability}

In $[5,8]$ we showed that only the "maximal" SPBs, i.e. those with $\tilde{N}$ spatial modes over a background with $\tilde{N}$ instabilities, are neutrally stable. In a sense, the maximal SPBs have saturated all the instabilities of the underlying plane wave. As an example, if the plane wave has only one UM, $b_{1}(x, t)$ is neutrally stable. On the other hand, $b_{1}(x, t)$ is unstable for any $L$ for which the underlying plane wave has $\tilde{N} \geq 2$ UMs.

In this section we provide numerical evidence that the rational solutions over the plane wave, $p_{i}(x, t)$, are linearly unstable. Given the interpretation of these rational solutions as singular limits of $b_{i}(x, t)$ as $L \rightarrow \infty$, this is consistent with the instability results for the SPBs.

To examine the stability of the rational solutions $p_{i}(x, t)$ we add a small perturbation to 
the initial condition at $t=0$,

$$
P_{i, \delta}(x, 0)=p_{i}(x, 0)+\delta f_{j}(x) .
$$

If $P_{i, \delta}(x, t)$ remains "close" to $p_{i}(x, t)$, in an appropriate sense as discussed below, then this indicates the solution is stable. However, if $P_{i, \delta}(x, t)$ diverges from $p_{i}(x, t)$ exponentially fast in time, then $p_{i}(x, t)$ is unstable. The initial data considered is a perturbation at the peak of the rogue wave $p_{i}(x, 0)$. In all the perturbed experiments, $\delta=10^{-2}$ and the time frame is $0 \leq t \leq 15$.

For each of the following perturbations $f_{j}(x), j=1, \ldots, 5$, an ensemble of 100 numerical experiments was carried out by varying the random component $\phi$ or $r_{k}(x) \in[0,1]$ in the initial data:

a) $f_{1}(x)=\cos 2 \pi k(x+\phi) / L, k=1,2$ where $\phi \in[0,1]$ is a random shift in the phase;

b) $f_{2}(x)=r(x)$ where $r(x) \in[0,1]$ is random noise;

c) $f_{3}(x)=\sum_{k=1}^{K} r_{k}(x) \mathrm{e}^{-\left(x-x_{k}\right)^{2}}$, representing $K$ localized Gaussian perturbations about $x_{k}$.

d) $f_{4}(x)=\sum_{k=l o w-m o d e s} r_{k}(x) \mathrm{e}^{i 2 \pi k x / L}$, representing a low frequency perturbation.

e) $f_{5}(x)=\sum_{k=h i g h-\text { modes }} r_{k}(x) \mathrm{e}^{i 2 \pi k x / L}$, representing a high frequency perturbation.
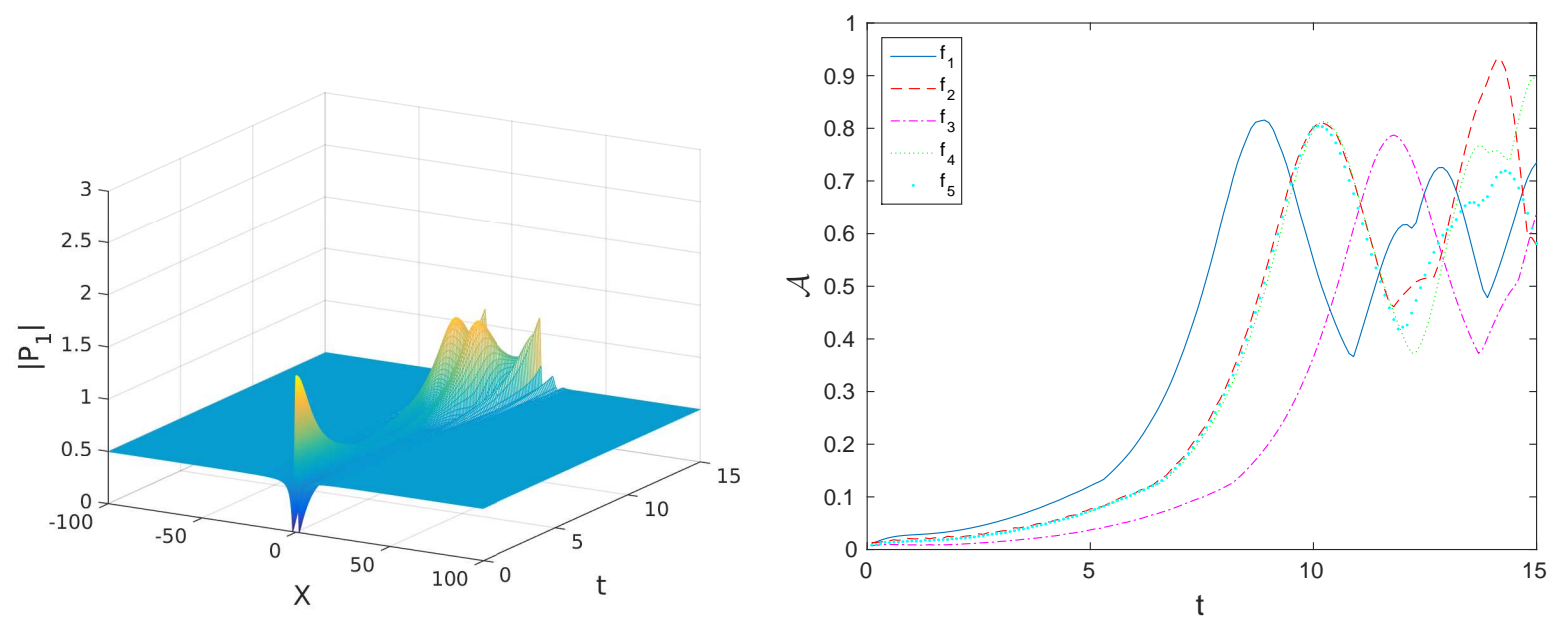

Figure 4: $P_{1, \delta}(x, t)$ experiments using CPS4 with $N=1024$ and $d T=2.36 \times 10^{-6}$ : (a) A sample surface for $P_{1, \delta}(x, 0)=p_{1}(x, 0)+\delta f_{3}(x)$ for $K=1$ and $x_{1}=0$. (b) Evolution of $\mathcal{A}_{j}(t)$ for each perturbation.

Since the rational solutions have steep gradients near their peak, we use the $H^{1}$-norm to address the issue of "closeness". Specifically, we examine the evolution of the difference of the perturbed solution, $P_{i, \delta}(x, t)$, and the nearest element of the unperturbed family of rational solutions, $p_{i}\left(x, t-t_{k}\right)$, which has the peak of the rogue wave at $t_{k}$ (rather than at $t=0$ as depicted in the earlier Figures). We define

$$
\mathcal{D}\left(t ; t_{k}\right)=\left\|P_{i, \delta}(x, t)-p_{i}\left(x, t-t_{k}\right)\right\|_{H^{1}},
$$



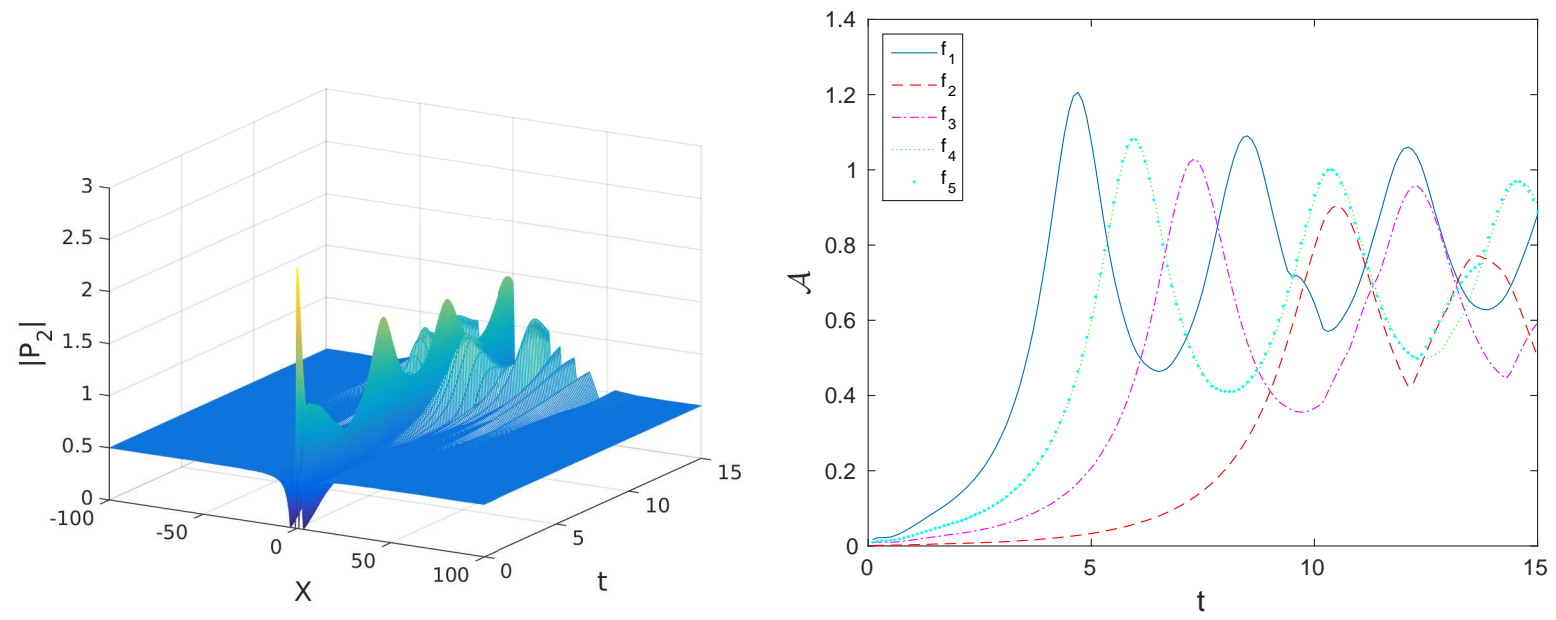

Figure 5: $P_{2, \delta}(x, t)$ experiments using CPS4 with $N=1024$ and $d T=2.36 \times 10^{-6}$ : (a) A sample surface for $P_{2, \delta}(x, 0)=p_{2}(x, 0)+\delta f_{4}(x)$. (b) Evolution of $\mathcal{A}_{j}(t)$ for each perturbation.

where

$$
\|u(x, t)\|_{H^{1}}=\int_{x_{1}}^{x_{N}}\left(\left|u_{x}\right|^{2}+|u|^{2}\right) d x
$$

and determine

$$
\mathcal{D}_{\max }\left(t_{k}\right)=\max _{t \in[0,15]} \mathcal{D}\left(t ; t_{k}\right)
$$

We then select the parameter value $t_{k}^{*}$ which minimizes $\mathcal{D}_{\max }\left(t_{k}\right)$, i.e

$$
\mathcal{D}_{m m}=\min _{t_{k}} \mathcal{D}_{\text {max }}\left(t_{k}\right)=\mathcal{D}_{\text {max }}\left(t_{k}^{*}\right)
$$

As such, $p_{i}\left(x, t-t_{k}^{*}\right)$ is the closest element of the family of first order $(i=1)$ or second order $(i=2)$ rational solutions and the evolution of $\mathcal{D}\left(t ; t_{k}^{*}\right)$ provides a measure of how close the perturbed solution is to an element of the rational family. An estimate of closeness for the ensemble is obtained using $\mathcal{A}_{j}(t)$, the average of $\mathcal{D}\left(t ; t_{k}^{*}\right)$ over all 100 simulations, for each $f_{j}(x)$ (note that $t_{k}^{*}$ is different for each simulation).

Figures 4(a) and 5(a) provide sample surfaces for perturbations $P_{1, \delta}(x, t)$ and $P_{2, \delta}(x, t)$, respectively. In Figures $4(\mathrm{~b})$ and $5(\mathrm{~b}), \mathcal{A}_{j}(t)$ grow exponentially fast to $\mathcal{O}(1)$ for all the random perturbations $f_{j}(x)$ considered, indicating $p_{i}(x, t)$ are linearly unstable, inheriting the instabilities of the underlying plane wave.

Observation: The rational solutions $p_{i}(x, t)$ of the NLS equation are linearly unstable since $P_{i, \delta}(x, t)$ diverge exponentially fast from $p_{i}(x, t)$ for $i=1,2$.

Considering robustness to be a measure of persistence of features of a solution, which is a different issue than stability, on a qualitative level the peak of the rational solutions appear robust. This is illustrated in Figures 6(a) - 6(b), where the numerical solution is initialized at $t=-10$, i.e. $P_{i}(x, 0)=p_{i}(x,-10)+\delta f_{j}(x)$. The rogue waves are clearly discernible. 

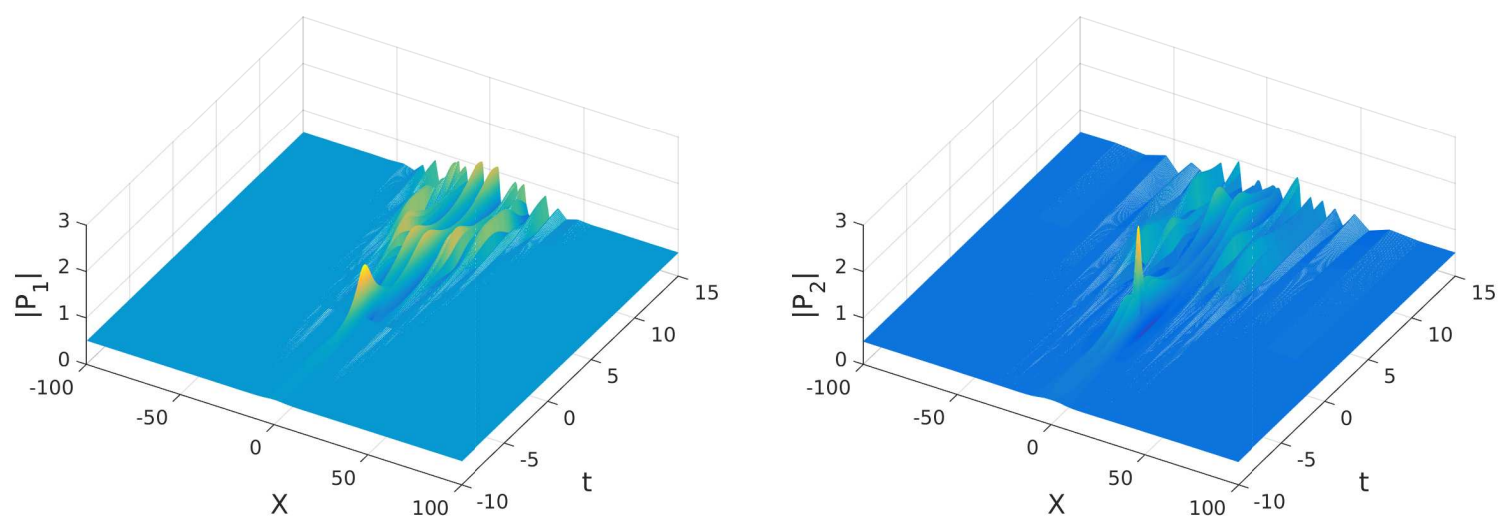

Figure 6: The surfaces for (a) $\left|P_{1, \delta}(x, t)\right|$ with $P_{1, \delta}(x, 0)=p_{1}(x,-10)+\delta f_{3}(x)$ : and (b) $\left|P_{2, \delta}(x, t)\right|$ with $P_{2, \delta}(x, 0)=p_{2}(x,-10)+\delta f_{4}(x)$.

\section{Resolving numerical errors associated with Fourier spectral methods}

In this section we discuss the numerical errors which arise using a pseudo-spectral scheme (FPS4) and which are representative of the results obtained using other Fourier integrators, e.g. the Fourier split step and exponential time-stepping integrators. Gibbs phenomenon yields oscillations which initially are tiny (e.g. $\left.\mathcal{O}\left(10^{-6}\right)\right)$, but grow to $\mathcal{O}(1)$ due to the linear instability of the Peregrine solution. In subsection 5.4 we adapt the FPS4 with a spectralsplitting method to reduce the Gibbs oscillations and improve the numerical solution.

In this section, since we work only with the periodically extended Peregrine solution, we drop the subindices and denote the periodic analytic and numerical Peregrine solutions by $p(x, t)$ and $P(x, t)$, respectively. The numerical results for the one-mode SPB are included in the discussion and used as a benchmark since the Peregrine solution is a limit of the SPB.

\subsection{Pseudo spectral method}

Let $u(x, t)$ be an exact solution of the NLS equation and $U(t)=\left(U_{1}(t), U_{2}(t), \ldots, U_{N}(t)\right)$ its numerical approximation at the grid points $X=\left(x_{1}, x_{2}, \ldots, x_{N}\right), x_{i}=-L / 2+i L / N$. Then, denoting the discrete Fourier transform and its inverse by $\mathcal{F}$ and $\mathcal{F}^{-1}$, we use the Fourier approximation

$$
u_{x x} \approx U_{x x}=\mathcal{F}^{-1}\left\{-k^{2} \mathcal{F}\{U\}\right\}, k=\frac{2 \pi}{L}[-N / 2+1, \ldots,-1,0,1, \ldots, N / 2]
$$

and a 4th-order in time Runge-Kuta scheme to solve the system of complex ordinary differential equations (8). We denote this 4 th-order in time pseudo-spectral scheme for solving the NLS equation as FPS4.

\subsection{Numerical results using FPS4}

Figure 7(a) shows the surface of the numerical Peregrine solution, $|P(x, t)|$, obtained with FPS4 using $L=200, N=2048$, and $d T=10^{-3}$. For contrast Figure $7(\mathrm{~b})$ shows the surface 
$|B(x, t)|$ for the SPB. The amplitude, location, and smooth behavior near the rogue wave are well resolved for both $P(x, t)$ and $B(x, t)$. However $P(x, t)$ develops spurious oscillations at the boundary which start to be visible at $t \approx 25$. For fixed $L$, there is little improvement in $P(x, t)$ as $N$ is increased and $d T$ is decreased, indicating there is a loss of regularity in the initial data.
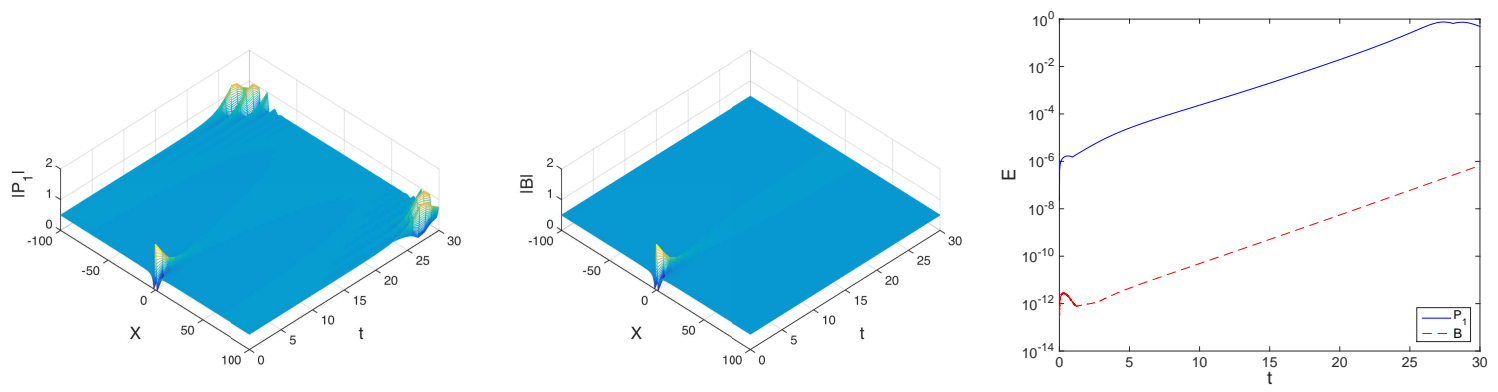

Figure 7: Numerical solution surfaces (a) $|P(x, t)|$, (b) $|B(x, t)|$ together with (c) the errors $E(t)$ for the Peregrine (blue solid) and for the SPB (red dashed) obtained with FPS4 for $L=200, N=2048$, and $d T=10^{-3}$.

Figure 7(c) plots the maximum errors in the numerical approximation of the Peregrine solution (blue solid line), now defined as $E(t)=\max _{x \in[-L / 2, L / 2]}(|| p(x, t)|-| P(x, t)||$ ), (and equivalently for the errors in the SPB (red dashed line)). The errors in the Peregrine solution are significantly larger than the errors in the SPB. A closer examination of the error in $P(x, t)$ shows that although the error is near round off at $t=0$, it abruptly jumps to $\mathcal{O}\left(10^{-6}\right)$ within the first few time steps and then grows exponentially to $\mathcal{O}(1)$ by $t \approx 25$. The exponential growth in the errors in the Peregrine solution provides further substantiation that it is unstable (as is the single mode SPB when considered on this large period).

\subsection{Source of the error}

Figure 8(a) shows the truncated and periodically extended Peregrine initial condition $P(x, 0)$ around $x=-L / 2=-80$. Although $P(x, 0)$ is continuous, since it decays agebraically
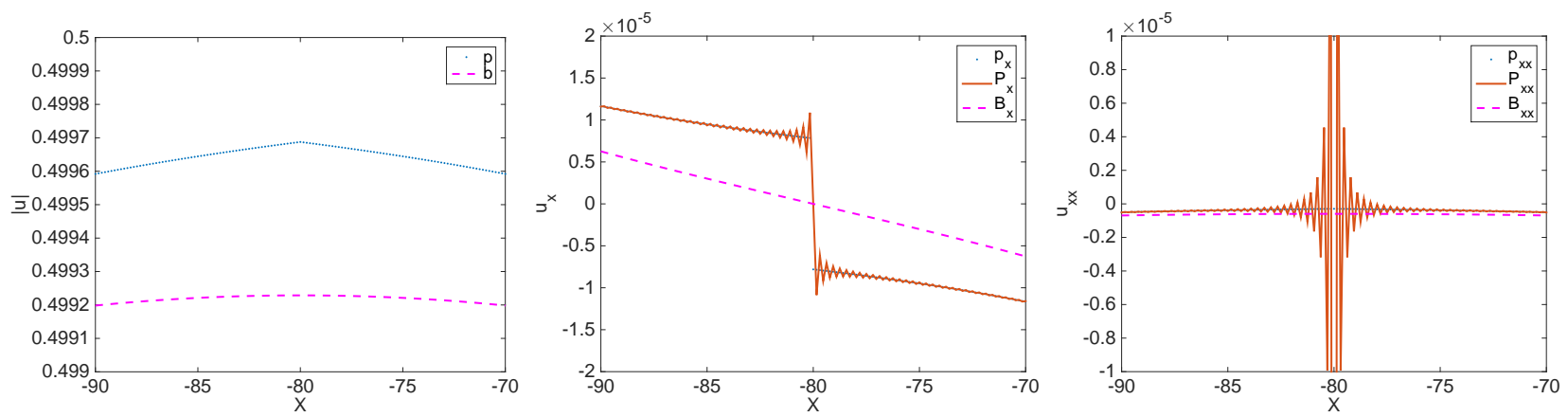

Figure 8: (a) $P(x, 0)$ together with the Fourier approximations of its (b) first and (c) second derivatives around the boundary point $x=-L / 2$. For comparison, $B(x, 0)$ and its derivatives (free of Gibbs oscillations) are shown in dashed lines. 
in space, there are almost unnoticeable corners at the boundaries. As a result, $P_{x}(x, 0)$ has a small jump discontinuity $\mathcal{O}\left(10^{-5}\right)$ at $x= \pm L / 2$ for $L=160$.

It is well known that the Fourier approximation of a function with a jump discontinuity exhibits Gibbs oscillations in the neighborhood of the discontinuity. This is shown in Figures 8(b) and 8(c) where oscillations overshooting the derivatives are evident in the Fourier approximations of both $P_{x}(x, 0)$ and $P_{x x}(x, 0)$. For comparison, the SPB initial condition $B(x, 0)$ and its derivatives (free of Gibbs oscillations) are shown in dashed lines.

The oscillations in $P(x, t)$ initially are not noticeable, since the size of the jump discontinuity in $P_{x}$ and error in $P_{x x}$ are very small (i.e. they are $\mathcal{O}\left(10^{-5}\right)$ and $\mathcal{O}\left(10^{-4}\right)$, respectively, for $L=200$ ). The overshoot depends on the magnitude of the jump discontinuity and it is not reduced by increasing the number of Fourier modes [14]. However, the jump is reduced by increasing the period $L$. Even so, the problem still persists (e.g. see Figure 11, where a larger spatial domain is used).

Significantly, the growth of the oscillations in $P(x, t)$ to $\mathcal{O}(1)$ provides further evidence of the linear instability of the Peregrine solution. For stable solutions the tiny errors introduced by Gibbs phenomenon on the order of e.g. $\mathcal{O}\left(10^{-5}\right)$ in the initial stage of the integration will not grow and are not sufficient to destroy a numerical solution. For example, consider perturbed soliton initial data of the form:

$$
U_{\delta}(x, 0)=\operatorname{sech}(x)+\delta\left(e^{-\left(x+x_{0}\right)^{2}}+e^{-\left(x-x_{0}\right)^{2}}\right),
$$

where $x_{0}=L / 2+\epsilon$ and $10^{-4} \leq \delta \leq 10^{-2}$. This initial data introduces a corner at the boundary, with an $\mathcal{O}(\delta)$ jump in the derivative, and subsequent Gibbs error in the Fourier approximations of the derivatives. Notice that this jump discontinuity is significantly larger than in the case of the periodically extended Peregrine solution. Using FPS4 to solve the NLS equation on the truncated domain $-50 \leq x \leq 50$ and time interval $0 \leq t \leq 100$ for $\delta=10^{-4}, 10^{-3}, 10^{-2}$, Figure 9 shows the perturbed solution $U_{\delta}(x, t)$ remains $\mathcal{O}(\delta / 10)$ close to $\operatorname{sech}(x)$ for $0 \leq t \leq 100$ as the Gibbs error does not grow.

\subsection{Reducing Gibbs oscillations with a modified FPS4}

Using FPS4 spurious $\mathcal{O}(1)$ oscillations develop in $P(x, t)$ as a result of Gibbs phenomenon coupled with the linear instability of the Peregrine solution. In this section we modify FPS4 using a spectral splitting technique (see e.g. [15]) that removes the Fourier modes associated with the Gibbs oscillations, leaving only those Fourier modes which contain the relevant information on the nature of the Peregrine solution.

The starting point of the proposed method is based on the following observation: a discontinuous derivative $u_{x}$ at a point $x_{d}$ can be expressed as the sum of two functions:

$$
u_{x}(x)=s(x)+v_{x}(x)
$$

where $s(x)$ is a discontinuous step function with a jump discontinuity of size $s_{0}=u_{x}\left(x_{d}^{+}\right)-$ $u_{x}\left(x_{d}^{-}\right)$at $x_{d}$ and $v_{x}(x)$ is a continuous function. The periodic assumption forces $s(x)$ to 

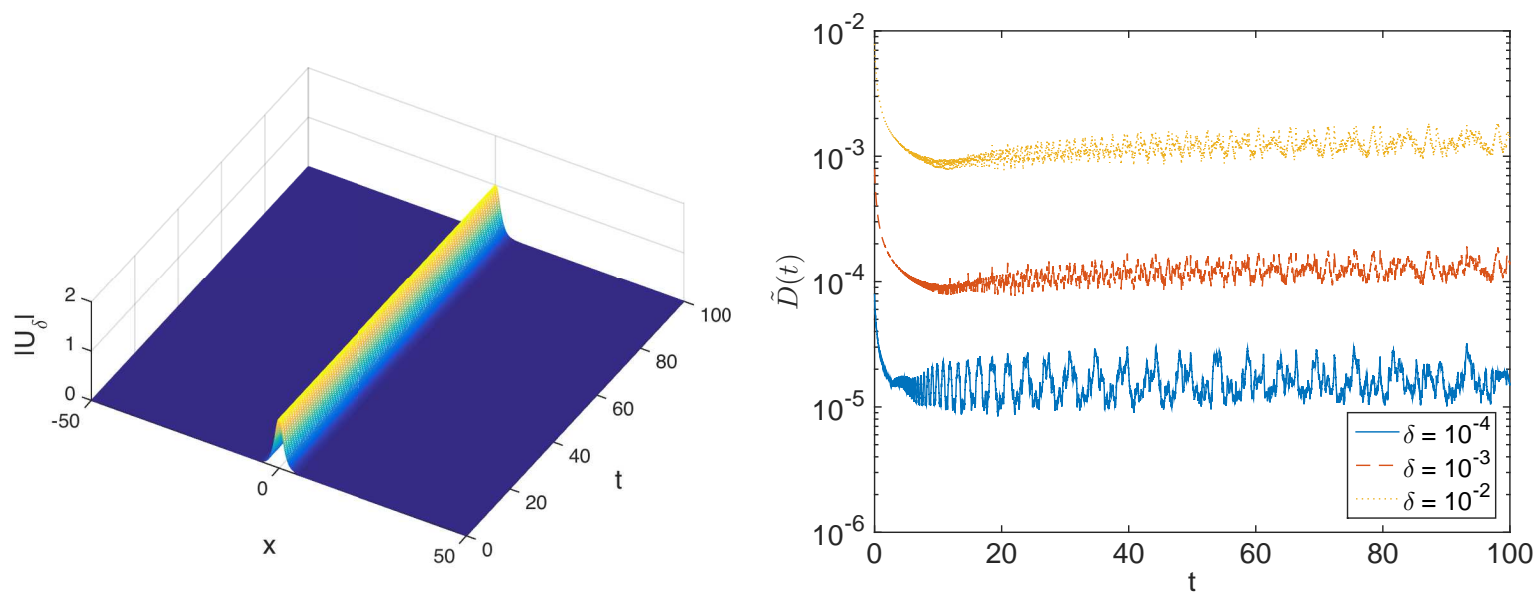

Figure 9: (a) The surface $\left|U_{\delta}\right|$ for $\delta=10^{-2}$, and (b) the difference $\tilde{D}_{\delta}(t)$ for perturbed soliton initial data with a corner at the boundary for $\delta=10^{-4}, 10^{-3}, 10^{-2}$.

have an additional jump discontinuity. Smoothing this second discontinuity and integrating we obtain $u(x)$ as

$$
u(x)=S(x)+v(x)
$$

where, both $S(x)$ and $v(x)$, are smooth continuous functions. This, together with the linearity of the Fourier transform $\mathcal{F}$, allows us to recover $u_{x x}$, without Gibbs oscillations, by means of

$$
u_{x x}(x) \approx S_{x x}+\mathcal{F}^{-1}\left\{-k^{2}(\mathcal{F} u-\mathcal{F} S)\right\}
$$

for a sufficiently smooth $S(x)$.

For example, considering

$$
S(x)=\frac{L}{\pi} P_{x}(-L / 2,0) \cos \left(\frac{\pi}{L} x\right)
$$

we apply this smoothing technique to $P(x, 0)=p(x, 0)$ with $L=200$ and $N=2048$. In Section 5.3, the Gibbs oscillations in the Fourier approximation of the first derivative $P_{x}(x, 0)$ are evident in Figure 8(b). Let $W$ denote the approximation of $P$ obtained using equations (18) and smoothing function $S(19)$. In Figure 10(a) $W_{x}(x, 0)$ is almost identical to the exact derivative $P_{x}(x, 0)$ and does not have oscillations around the jump discontinuity. Figures $10(\mathrm{~b}-\mathrm{c})$ show that the errors in approximating $P_{x}(x, 0)$ and $P_{x x}(x, 0)$ with $W_{x}$ and $W_{x x}$ are $\mathcal{O}\left(10^{-13}\right)$ and $\mathcal{O}\left(10^{-12}\right)$, respectively for $-L / 2<x<L / 2$ (the error in approximating $P$ with $W$ is $\mathcal{O}\left(10^{-16}\right)$, as expected).

The effect of using the spectral splitting technique on the numerical solution is shown in Figures 11(a-c), for $L=800, N=8192$, and $d T=2.5 \times 10^{-5}$. Figure 11(a) shows the original surface $|P(x, t)|$ obtained without any smoothing. The surface $|W(x, t)|$ (Figure 11(b)) shows that the smoothing function (19) significantly reduces the spurious oscillations. Figure 11(c) provides the error $E(t)$ in the Peregrine solution $p(x, t)$ when using numerical solution $P(x, t)$ (with no smoothing - solid line) and solution $W(x, t)$ (with smoothing function $S$ - dashed 

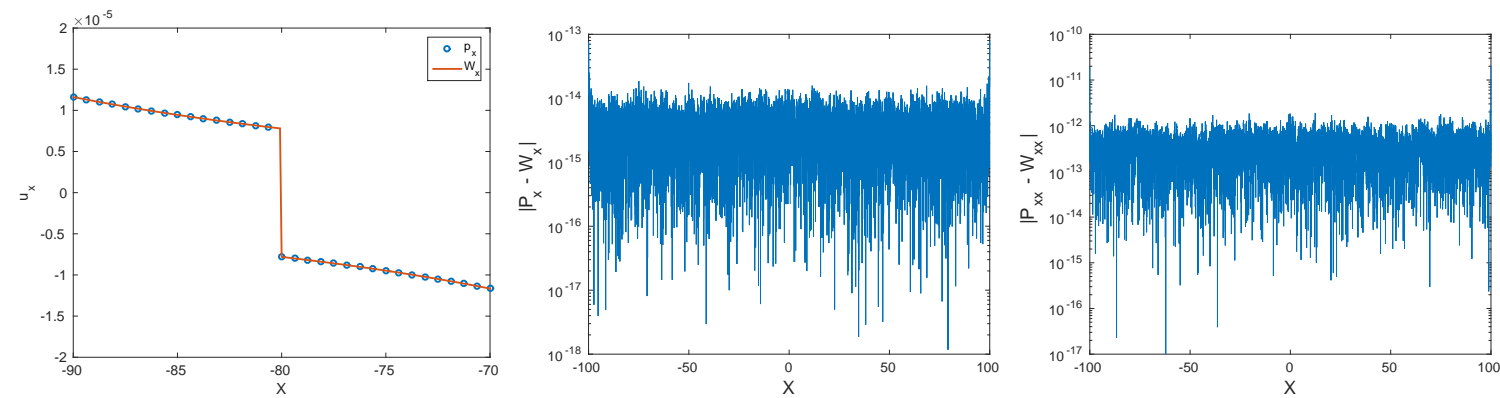

Figure 10: (a) $W_{x}(x, 0)$, the approximation of $P_{x}(x, 0)$ without Gibbs oscillations. The errors in (b) $P_{x}(x, 0)$ and (c) $P_{x x}(x, 0)$ for $L=200, N=2048$. ( $W$ is the approximation to $p$ using $S$ ).
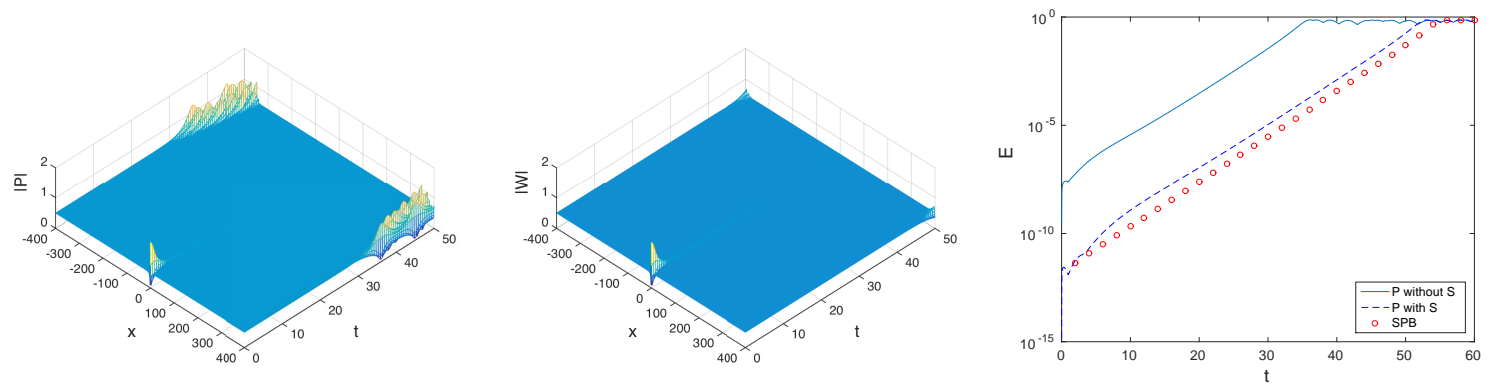

Figure 11: Numerical Peregrine solution surfaces: (a) $|P(x, t)|$ with no smoothing and (b) $|W(x, t)|$ with smoothing function $S$ together with (c) their errors $E(t)$ obtained with FPS4 for $L=800, N=8192$, and $d T=2.5 \times 10^{-5}$. 
line). The error in the SPB solution obtained with FPS4 is used as a benchmark (o-line). $P(x, t)$ reaches $\mathcal{O}(1)$ at $t \approx 36$, while $W(x, t)$ reaches $\mathcal{O}(1)$ at about $t \approx 52$. In contrast, the error in the SPB reaches $\mathcal{O}(1)$ at about $t \approx 56$. The numerical solutions still suffer from the growth of roundoff error, which is inherent, since both solutions are unstable.

In summary, we developed a highly accurate Chebyshev psuedo-spectral method useful in studying infinite line problems and showed the rational solutions of the NLS equation are linearly unstable. To mitigate the errors associated with standard Fourier methods we developed a technique that resolves Gibbs oscillations due to discontinuous data for the NLS equation. The modified FPS4 method can treat initial data with discontinuous derivatives over periodic domains, of which the resolution of the Peregrine solution is a particular example.

\section{Acknowledgments}

The authors gratefully acknowledge the support of the National Science Foundation, Grant DMS-1108973.

\section{References}

[1] N. Akhmediev, A. Ankiewicz, J.M. Soto-Crespo, Rogue waves and rational solutions of the nonlinear Schrödinger equation, Phys. Rev. E 80 (2009) 026601

[2] N. Akhmediev, A. Ankiewicz, M. Taki, Waves that appear from nowhere and disappear without a trace, Phys. Lett. A 373 (2009) 675-678

[3] J.P. Boyd, Spectral methods using rational basis functions on an infinite interval, J. Comp. Phys. 69 (1987) 112-142

[4] A. Calini, C.M. Schober, Homoclinic chaos increases the likelihood of rogue waves, Phys. Lett. A 298 (2002) 335-349

[5] A. Calini, C.M. Schober, Observable and reproducible rogue waves, J. Optics, 15, 105201, (2013).

[6] K.B. Dysthe, K. Trulsen, Note on breather type solutions of the NLS as models for freak-waves, Physica Script T82 (1999) 48-52

[7] P. Gaillard, Families of quasi-rational solutions of the NLS equation and multi-rogue waves, Journal of Physics A: Math and Theor, 44, (2011)

[8] A. Calini, C.M. Schober, Numerical investigation of stability of breather-type solutions of the nonlinear Schrödinger equation, Nat. Hazards Earth Syst. Sci. 14 (2015) 14311440

[9] A. Calini, C.M. Schober, Dynamical criteria for rogue waves in nonlinear Schrödinger models, Nonlinearity 25 (2012) 99-116 
[10] A. Osborne, M. Onorato, M. Serio, The nonlinear dynamics of rogue waves and holes in deep-water gravity wave trains, Phys. Lett. A 275 (2000) 386

[11] D.H. Peregrine, Water waves, nonlinear Schrödinger equations and their solutions, J. Aust. Math. Soc. B 25 (1983) 16-43

[12] D.H. Sattinger, V.D. Zurkowski, Gauge theory of Bäcklund transformations, PhysicaD 26 (1987) 225

[13] L. Shemer, L. Alperovich, Peregrine breather revisited, Phys. Fluids 25 (2013) 051701

[14] L.N. Trefethen, Spectral Methods in MATLAB SIAM ISBN 0-89871-465-6, 2000.

[15] J.G. Wangüemert-Perez, R. Godoy-Rubio, A. Ortega-Moñux, and I. Molina-Fernandez, Removal of the Gibbs phenomenon and its application to fast-Fourier-transform-based mode solvers, J. Opt. Soc. Am. A 24 (2007) 3772-3780

[16] G. Yang, Y. Wang, Z. Qin, B.A. Malomed, D. Mihalache, L. Li, Breather-like solitons extracted from the Peregrine rogue wave, Phys. Rev. E 90 (2014) 062909

[17] V.E. Zakharov, A.B. Shabat, Exact theory of two-dimensional self-focusing and one-dimensional self-modulation of waves in nonlinear media, Journal of Experimental and Theoretical Physics 34 (1972) 62

[18] B. Kibler, K. Hammani, C. Michel, C. Finot, A. Picozzi, Rogue waves, rational solitons and wave turbulence theory, Phys. Lett. A 375 (2011) 3149-3155

[19] D. Gottlieb, C.W. Shu, On the Gibbs phenomenon and its resolution, SIAM Rev. 39 (2005) 644-668

[20] J. Garnier, K. Kalimeris, Inverse scattering perturbation theory for the nonlinear Schrödinger equation with non-vanishing background, J. Phys. A: Math. Theor. 45 (2012) 035202

[21] A.V. Slunyaev, V.I. Shrira, On the highest non-breaking wave in a group: fully nonlinear water wave breathers vs weakly nonlinear theory, J. Fluid Mech. 735 (2013) 203

[22] A. Slunyaev, E. Pelinovsky, A. Sergeeva, A. Chabchoub, N. Hoffmann, M. Onorato, N. Akhmediev, Super rogue waves in simulations based on weakly nonlinear and fully nonlinear hydrodynamics equations, Phys. Rev. E 88 (2013) 012909 